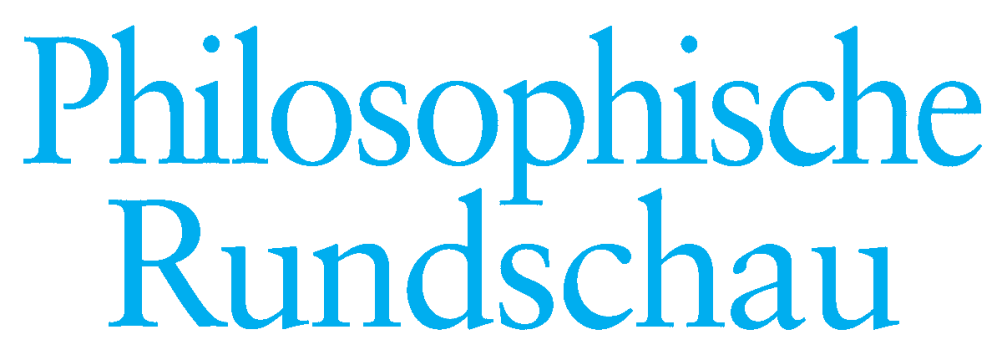

Eine Zeitschrift für philosophische Kritik

Band 62 Herausgegeben von

Heft 3 Jens Halfwassen - Pirmin Stekeler-Weithofer

2015 Bernhard Waldenfels

Antje Kapust Sprachen der Ikonizität.

Neuere Ansätze in der Bildforschung 191-224

Felix Heidenreich Politik der Wahrheit,

Wahrheit der Politik. Was ist neu am

»Neuen Realismus«? 225-242

Burkhard Liebsch Spielräume einer Kultur

der Gastlichkeit. Zwischen Ethik, Recht und Politik

(2. Teil) $\quad \mathbf{2 4 3 - 2 6 0}$

Ingo Meyer Neues aus der Carl Schmitt-

Industrie 261-273

Andreas Urs Sommer Machtspiele 274-276 


\section{Philosophische Rundschau}

Eine Zeitschrift für philosophische Kritik

\section{Redaktion:}

Prof. Dr. Martin Gessmann, Hfg-Offenbach am Main, Schloßstr. 31, 63065 Offenbach/ Main, Telefon: 069 /80059-164, e-mail: Gessmann@hfg-offenbach.de

Die Philosophische Rundschau veröffentlicht ausschließlich deutschsprachige Artikel, die in der Regel vorher mit der Redaktion abgesprochen werden.

Mit der Annahme zur Veröffentlichung überträgt der Autor dem Verlag das ausschließliche Verlagsrecht für die Publikation in gedruckter und elektronischer Form. Weitere Informationen dazu und zu den beim Autor verbleibenden Rechten finden Sie unter www.mohr. de/phr.

Ohne Erlaubnis des Verlags ist eine Vervielfältigung oder Verbreitung der ganzen Zeitschrift oder von Teilen daraus in gedruckter oder elektronischer Form nicht gestattet. Bitte wenden Sie sich an rights@mohr.de.

Im Abonnement für Institutionen und Privatpersonen ist der freie Zugang zum OnlineVolltext enthalten. Institutionen mit mehr als 20.000 Nutzern bitten wir um Einholung eines Preisangebots direkt beim Verlag. Kontakt: elke.brixner@mohr.de. Um den OnlineZugang für Institutionen / Bibliotheken einzurichten, gehen Sie bitte zur Seite: www.in gentaconnect.com/register/institutional. Um den Online-Zugang für Privatpersonen einzurichten, gehen Sie bitte zur Seite: www.ingentaconnect.com/register/personal.

(C) 2015 Mohr Siebeck GmbH \& Co.KG, Tübingen. - Die Zeitschrift und alle in ihr enthaltenen einzelnen Beiträge und Abbildungen sind urheberrechtlich geschützt. Jede Verwertung außerhalb der engen Grenzen des Urheberrechtsgesetzes ist ohne Zustimmung des Verlags unzulässig und strafbar. Das gilt insbesondere für Vervielfältigungen, Übersetzungen, Mikroverfilmungen und die Einspeicherung und Verarbeitung in elektronischen Systemen.

Satz und Druck: Gulde-Druck, Tübingen; Bindung: Nädele, Nehren.

ISSN 0031-8159 


\title{
Neues aus der Carl Schmitt-Industrie
}

\author{
Ingo Meyer
}

Stefan Breuer: Carl Schmitt im Kontext. Intellektuellenpolitik in der Weimarer Republik. Berlin 2012. Akademie Verlag. 303 S.

Jean-François Kervégan: Que faire de Carl Schmitt? Paris 2011. Gallimard. 331 S. Carl Schmitt: Der Schatten Gottes. Introspektionen, Tagebücher und Briefe 1921 bis 1924. Hg. v. Gerd Gieseler, Ernst Hüsmert und Wolfgang H. Spindler. Berlin 2014. Duncker \& Humblot. 601 S.

\section{I}

Manchen provoziert Carl Schmitt noch immer, ${ }^{1}$ da ist es oftmals hilfreich zu beobachten, wie die französischen Nachbarn mit unseren berüchtigteren Denkern umgehen. Jean-François Kervégan allerdings ist voller Bewunderung für den deutschen rechtsphilosophischen Diskurs des frühen 20. Jahrhunderts insgesamt: »Il me paraît clair que l'ensemble des problemes de la philosophie du droit a à prendre en charge ne saurait être traité de manière satisfaisante dans l'horizon qui était celui des débats allemands de la première moitié du XX $\mathrm{X}^{\mathrm{e}}$ siècle« (75).

Der Autor, geb. 1950, lehrt Philosophie an der Universität Paris I-Panthéon-Sorbonne, zählt zur letzten Alterskohorte der vom Marxismus enttäuschten französischen Linksintellektuellen, bewegte sich zum Hegel des objektiven Geistes ${ }^{2}$ und entdeckte bei damals noch dürftiger Übersetzungslage um 1980 auch Carl Schmitt, was bereits in Kervégans Habilitationsschrift zum Versuch führte, beide produktiv aneinander zu spiegeln. ${ }^{3}$

Auch angesichts der zunehmenden Anzahl von Übersetzungen Schmitts ins Französische erhebt Que faire de Carl Schmitt? doppelten Anspruch, eine nicht zu knappe Einführung mitsamt der Erläuterung von Grundbegriffen für das französische Publikum zu liefern, sowie einen pragmatischen Umgang mit dem Ideengut nahezulegen: Im Ausgang von und nach Carl Schmitt das Politische zu denken (73ff.), das liegt ganz fraglos im Trend.

Das Buch zerfällt in zwei Teile, eine kontextualisierte Werkpräsentation in Kurzform und einen weitaus längeren Abschnitt, der zentrale Kategorien

\footnotetext{
${ }^{1}$ Jüngst, im Ton durchgängiger Empörung, aber ohne jede neue Erkenntnis Dirk HoEGES: Die Menschenrechte und ihre Feinde. Deutsche Profile zwischen Kaiserreich und Bundesrepublik, Köln 2013, S. 30, 58f., 76ff., 199ff. u. passim.

2 Jean-François Krevégan: L'effectif et le rationnel. Hegel et l'esprit objectif, Paris 2008.

3 Jean-François Krevégan: Hegel, Carl Schmitt. La politique entre spéculation et positivité, Paris 1992.
} 
Schmitts recht ausführlich verhandelt: Theologie, Normativität, Legitimität, Politik und /Weltr.

Die Eröffnung bringt für den deutschen Leser wenig Überraschendes, die "contradiction aiguë entre rationalisme et irrationalisme qui traverse toute la pensée de Carl Schmitt" wird ebenso bündig benannt (37) wie Kervegán um die Frage, ob Schmitts Werk im Kern nazistisch zu veranschlagen oder sein Engagement nur eine Verirrung gewesen sei, inklusive Vorschlägen zu Binnendifferenzierungen, großen Aufwand betreibt - um dann doch die Waffen zu strecken. Das Problem bleibe "son arcanum, son art secret", aber es lasse sich durchaus mit Konzepten arbeiten, die innerhalb eines unerfreulichen Rahmens entwickelt worden seien (64). Eben. Kurze Übersichten zur Wirkung speziell in Deutschland, Frankreich und Italien, mitsamt der Rekonstruktion von Ellen Kennedys Skandalthese von 1986, die Frankfurter Schule, insbesondere Habermas' Frühwerk, speise sich nicht unwesentlich auch aus Motiven Schmitts, ${ }^{4}$ runden den ersten Teil ab.

Der Einstieg in die Begriffsentfaltung mit der politischen Theologie gerät ein wenig umständlich; Säkularisierungs- und Neutralisierungsthese, Repräsentationskonzept, totaler Staat, die Esoterik des paulinischen, von Schmitt anverwandelten Katechon und die Implikationen der Geschichtsmetaphysik werden erörtert, der Beziehung der ersten Politischen Theologie von 1922 zur zweiten aus dem Jahre 1970 aber widmet Kevégan vielleicht doch zu viele Seiten. Ähnlich benötigt die Frage danach, wie man Schmitts Zugriff überhaupt bezeichnen könne, einen gar zu langen Anlauf, bis ihn der Autor endlich als "theologien politique" fasst (107), der jenseits von Bekenntnisfragen mit kaltem Blick nach der Korrelation von Staatsform und der sie überwölbenden Semantik fragt, ja der Ungeheuerlichkeit, dass überhaupt Staat ist, nachgeht. Mit Verlaub, all das findet man etwa bei Norbert Bolz auf wenigen Seiten gedrängter, auch präziser. ${ }^{5}$

Sehr viel eingängiger dagegen ist die schon in Kervégans Habilitation vorgetragene und hier noch einmal verfeinerte Konfrontation Schmitts mit Hans Kelsen als dem Hauptverteter des normativen Rechtspositivismus. Obwohl beide die aporetischen Untiefen aller Begründungsversuche des Rechts gesehen haben (131), unterlaufe Schmitts Dezisionismus Dualismen wie Norm und Kausalität, Chaos und Ordnung, Sein und Sollen und erscheint gegenüber Konstruktionen wie der »Grundnorm« mit ihrem zwangsläufig infiniten Regress und Stütztheoremen etwa des pyramidalen Stufenbaus der Rechtsordnung klar im Vorteil, auch wenn Kervégan im Anschluss an Giorgio Agamben die Gefahr einer entfesselten "politique pure, dégagée du droit« (141) erinnert, die dem Dezisionismus, der schon in Schmitts Gesetz und Urteil von 1912 festgemacht wird (127f.), fraglos inhärent ist. Ähnlich sicher stellt Kervégan im Rahmen der Legalitätsfrage Schmitts erstmals in der Parlamentarismusschrift gegebenen Hinweis auf die explizite Eklusivität von Demokratien und die implizite Asymmetrie

4 Ellen Kennedy: "Carl Schmitt und die ’Frankfurter Schule»", in: Geschichte und Gesellschaft 12 (1986), S. 380-419.

5 Norbert Bolz: Auszug aus der entzauberten Welt. Philosophischer Extremismus zwischen den Weltkriegen, München 1989, S. $53 \mathrm{ff}$. 
der vermeintlichen Chancengleichheit von Parteien im Parlamentarismus heraus: Wer hier überhaupt dazugehört und über legale, auf Mehrheiten gegründete Macht verfüge, habe stets mehr als diese Macht, nämlich in letzter Konsequenz die Möglichkeit, die Bedingungen der Legalität selbst $\mathrm{zu}$ definieren, es ergebe sich »une situation de concurrence inégale entre les partis« (150). Erinnert man Begriffe unseres politischen Systems wie ২Kanzlerbonus`, ‘Fünf-Prozent-Hürde` und `Verfassungsfeinde`, wird deutlich, dass man gar nicht in die radikalisierten Jahre der Weimarer Spätzeit zurückgehen muss, um zu sehen, dass Schmitt auch heute noch Bedenkenswertes zu liefern vermag. Kervégan schließt mit Ausführungen zum Legitimitätsbegriff der Schmitt-Lektüren Blumenbergs, Habermas' und Böckenfördes.

Die landläufig inkriminierte Definition des Politischen als "Unterscheidung von Freund und Feind " situiert Kervégan zunächst historisch, insofern wohltuend, als gerade in Frankreich Der Begriff des Politischen lange Zeit die einzige übersetzte Schrift Schmitts war (175); Nationalismus, Versailler Vertrag und Völkerbund als auch der Marxismus leninistisch-maoistischer Prägung, dem das Freund/Feind-Denken ebenfalls keineswegs fern stehe (181), sind die Eckdaten, aus denen Kervégan Schmitts Politisches zunächst herleitet, um es dann von irrigen Substanzannahmen, pessimistischer Anthropologie u.ä. zu befreien, handele es sich doch um eine spezifische Perspektive, Explikation der Logik eines Aktionsraumes quer zu Institutionen oder Funktionssystemen, "une conception énergétique«: »Si l'analyse schmittienne du politique a un intérêt actuel, c'est probablement dans cette direction non essentialiste, dynamique, qu'il est à chercher" (185f.). So aber gelte i.S. Schmitts nicht nur, dass es kein ıreines`, politikfreies Recht je geben kann, sondern auch, dass keinerlei Rechtsgut revisionsimmun ist: "Le politique, c'est l'acte de l'institution toujours révocable du juridique et non pas son autre ou son `environnement« (193). Wer bei Kervégan noch immer den langen Schatten eines ehemals ideologiekritischen Interesses erkennt, liegt sicher nicht ganz falsch.

Behutsam abwägend wird zum Ende des Durchgangs die Perspektive auf globale Relationen geöffnet, nicht ohne die Spekulationen über das Ende der Epoche klassischer Staatlichkeit und neue Formen der Integration in Schmitts Spätwerk historisch-genetisch nachzuweisen, er tritt hier erneut hellsichtiger auf als die Verfechter von Utopien einer via humanistischer Ideale angeleiteten, unifizierenden 'Weltpolitik (241f.).

Eine knappe Bilanz, "Le dissentiment«, beschwört noch einmal Voraussetzungen der Verfassung, drohende Kontamination von Politik und Moral, Bewusstsein für die stets brüchige Stabilisierungsfunktion des Rechts, Selbstwidersprüche der Moderne etc. als von Schmitt in ihrer Virulenz aufgezeigten Problemfelder. Betont Kervégan, dass man nicht den Antworten beipflichten müsse, um doch die "fécondité des concepts schmittiens" zu würdigen (250), verortet sich der Autor, kaum überraschend, deutlich als Linksschmittianer. Kervégan hat trotz mancher Länge sein 
Klassenziel erreicht, es sollte im gallophonen Sprachraum fortan schwerer fallen, Schmitt noch immer als Betriebsunfall juridischen Denkens oder gar direkten Abgesandten des Antichristen zu fliehen.

\section{II}

Der Hamburger Soziologe Stefan Breuer arbeitet sich mit bewunderungswürdiger Ausdauer seit gut zwei Jahrzehnten durch den Textcorpus des um 1870/71 mit neuer Vehemenz einsetzenden konservativen Denkens und kann auf eine stattliche Anzahl von Monographien zum überaus schillernden Themenspektrum zurückblicken. Carl Schmitt im Kontext nun gibt, anders als es der für Einführungen typische Titel vermuten lässt, nichts dergleichen, sondern versucht, konträr zu Positionen, die Schmitts Sprunghaftigkeit und Anpassungsbereitschaft, wenn nicht Unzuverlässigkeit akzentuieren, einen konsequenten Weg Schmitts in die politische Theorie der Moderne zu beschreiben. Auch deshalb beschränkt sich der Autor auf die Weimarer Jahre; einerseits geschieht dies zu Recht, da man wohl der Meinung sein kann, dass Schmitt speziell während der Bonner Lehrtätigkeit seine beste Zeit hatte $;^{6}$ andererseits ist dies schmerzlich, weil das eigentliche 'Skandalon Schmitt<, seine wenigstens bis zum Karriereknick von 1936 unstrittige Rechtfertigung des NS-Regimes nonchalant ausgeblendet wird: "Die Frage, ob Carl Schmitt und seine Schüler zu den Propagandisten und Apologeten des Völkermords gehörten, bezieht sich auf eine Phase, die außerhalb des hier gewählten Untersuchungszeitraums liegt und muß daher an dieser Stelle unbeantwortet bleiben" (9). Für ein Buch, das qua Titel Wert auf Kontexte und die interventionistische Potenz von Ideen legt, ist das eigentlich befremdlich.

Die gelinde methodische Polemik gegen den whermeneutische[n] $\mathrm{Zu}-$ griff» (8), den Breuer offenbar noch immer auf der Suche nach einem objektiv-zeitenthobenen Sinn wähnt, halte ich für gegenstandlos. Sein Ansatz dagegen präferiere "den subjektiv gemeinten Sinn, und das in strikt historischer und kontextbezogener Einstellung" (ebd.). Allein, war nicht genau das das hermeneutische Geschäft von Schleiermacher bis Hans Robert Jauß, was immer die Kritik der verkappten Werkästhetik des Letzteren angetragen haben mag? - De facto praktiziert Breuer denn auch nichts anderes als ganz unspektakuläre Hermeneutik, er rekonstruiert über weite Strecken Schmitts "Geistergespräch" mit dem gerade in diesem Fall überaus bunten Kosmos der »Ideengeschichte« (ebd.).

Die griffig und flott geschriebene Eröffnung skizziert Schmitts frühe Münchner Jahre der "Partizipation am Bohemeleben« (25), hier lernte er die linke Avant-

${ }^{6}$ Reinhard Mehring: Carl Schmitt. Aufstieg und Fall, München 2009, S. 142. 
garde und Anarchoszene hassen, was zu seinem für Breuer gar nicht zu überschätzenden Wunsch nach "Aufhebung der Entzweiung" und "Wiederentdeckung der Kirche« (32f.) geführt habe, liege an dieser Stelle doch das eigentliche Initial der katholizistisch gefärbten Substanzphantasien "gegen das Auseinandertreten von Denken und Sein" (38). Damit ist Schmitt als wesentlich philosophischer Denker installiert.

Auf eine Geduldsprobe stellt den Leser das Folgekapitel. Die sehr detaillierte Aufarbeitung von Schmitts Deutung des Abbé Sieyès, so wichtig sie als formativer Impuls für die Entwicklung von Schmitts Denken auch sein mag, das sich das Konzept der "souveränen pouvoir constituant a anverwandelt, um die voluntaristische Selbstsetzung der Nation zu denken (53), liest sich außerordentlich zäh und basiert zudem auf einem bereits 1984 publizierten Aufsatz Breuers, der hier Anlass zur Revision gibt. Versöhnt wird der Leser dann aber mit den Ausführungen zu Schmitts konstanter Wertschätzung des italienischen Faschismus, der ihm »eine Fortsetzung des Risorgimento-Nationalismus« schien (67), sodass der unmittelbare Zeitbezug wieder hergestellt ist.

Die Konfrontation mit Max Weber dagegen mutet sehr viel konzentrierter an. Breuer zeigt als profilierter Weber-Kenner, wie Schmitt unterhalb der "Höflichkeitsfloskeln« mittels des eigenen Ansatzes versucht, das Werk des soziologischen Übervaters "zu unterminieren« (83f.) und überhaupt dazu neigt, Soziologie in Metaphysik aufzulösen (100). Der Kontrast von Herrschaftsformen, Politik-, Rechts- und Staatsauffassung, all das ist präzise ausgeführt und wird mit einem Seitenblick auf den von Schmitt verehrten Ferdinand Tönnies gewürzt (95ff.), ohne dass man in der Summe doch grundstürzend Neues erführe. Trotz der vermeintlichen Nähe beider, etwa über die polemogene Semantik von Politik als Handlungsfeld des `Kampfes`, benennt Breuer klar die letztinstanzliche Unvereinbarkeit beider Ansätze.

Dass hier, abgesehen davon, dass Schmitt Hitler nie als Charismatiker wahrnehmen konnte, noch einmal die m.E. zu Tode diskutierte charismatische Herrschaft Webers aufgenommen wird, verblüfft besonders deshalb, weil sie für Breuer überraschend umstandslos mit moderner Demokratie kompatibel scheint, als hätte M. Rainer Lepsius nie ihren dispositionalen Kern als Bereitschaft zum Wunderglauben isoliert und sie Weber selbst als Herrschaftsform wesentlich der Vergangenheit gefasst - was gut so sei. ${ }^{7}$ Die zentrale Frage, ob dieses Herrschaftskonzept von Weber zu Schmitt das NS-Regime antizipierte und damit nicht zuletzt legitimierte, wird über den Rekurs auf die betagte Debatte zwischen Karl Löwith, Wolfgang J. Mommsen und Karl Löwenstein aus den frühen sechziger Jahren serledigt‘ $(101,107)$. Wenn also tatsächlich diese späte Kategorie Webers "die eigentliche, zeitgenössische Herausforderung ${ }^{8}$ an Schmitt darstellte, hätte

7 M. Rainer Lepsius: "Das Modell der charismatischen Herrschaft und seine Anwendbarkeit auf den >Führerstaat Adolf Hitlers«, in: ders.: Demokratie in Deutschland. Soziologisch-historische Konstellationsanalysen, Göttingen 1993, S.95-118, hier: 117; MAX WeBER: Brief an Arthur Salz vom 15./22. Februar 1912, in: ders.: Gesamtausgabe II/7: Briefe 19111912, hg. v. M. Rainer Lepsius u.a., Tübingen 1998, S. 429.

8 So wiederum schon Bolz, a.a.O., S. 81. 
man sich zumindest Hinweise auf neuere Literatur gew ünscht. ${ }^{9}$ Dafür arbeitet Breuer Schmitts auf theologisch-metaphysische Wurzeln zurückgehenden Begriff der (personalen) Repräsentation als Wiederherstellung oder Visualisierung eines substanziellen Seins in Differenz zum ımodernen Delegationsprinzip der Interessenvertretung, wie er m.W. erstmals im Essay Römischer Katholizismus und politische Form von 1923 entwickelt wurde, deutlich heraus (103ff.), folglich ein für künftige Belange im Seminarbetrieb doch recht kompakter Abschnitt entstanden ist, der auch gut für sich allein stehen könnte.

Überhaupt die Gliederung des Buches: Die neun materialen Kapitel hinterlassen das Gefühl, als öffne und schließe sich jeweils der Bühnenvorhang vor Schmitts Nachdenken, eine kontinuierliche narratio wurde von Breuer offenbar gar nicht erst beabsichtigt. Das ist bei der sachlichen Heterogenität gerechtfertigt, geht aber deutlich zu Lasten der monographischen Kohärenz, zumal das Buch erst jetzt, nach einem guten Drittel seines Umfangs, in der Rekonstruktion dessen, was man auch rambivalente Begegnungen nennen könnte, Drive entwickelt.

Die Einlassungen zu Schmitt »im Dialog« mit den jüdisch-sozialistischen Juristen Otto Kirchheimer, Franz Neumann und Ernst Fraenkel, letzterer noch bekannt durch seine Unterscheidung von Maßnahmen- und Normenstaat, sind schon insofern ein wichtiges Stück Ideengeschichte, als hier die Ursituation des Linksschmittianismus plastisch wird und bei aller Differenz im Einzelnen nach wie vor schmittianische Problemfelder wie die `Enthegung` des Krieges, die Gefahr einer Pervertierung des juridischen Rationalismus und die Reichweite des Staates überhaupt angelegentlich sind. Der gemeinsame Nenner aller vier von Breuer fokussierten Autoren ist denn auch Schmitts zentrale, doch höchst problematische Überzeugung einer Unverträglichkeit von »Demokratie und bürgerlich-liberaler Ordnung", die zu verschiedenen Diagnosen einer Erosion des Staates führten $(120,127)$.

Was nun folgt, ist aus Breuers stupender Quellenkenntnis, die auch diverse Nachlässe einbezieht, geschöpft: die Darstellung von Schmitts Kontakten zu verschiedenen, heute beinahe vergessenen konservativen Diskussionszirkeln und ihrer publizistischen Organe wie Die Tat, Der Ring und Deutsches Volkstum. Hier zeige sich »Intellektuellenpolitik« in actu: Die Schmittrezeption der federführenden Tat-Redakteure unter Hans Zehrer, ab 1929 elitär-jungkonservativ ausgerichtet, beruhe "weitgehend auf Mißverständnissen und Fehldeutungen" (151), zu stark nämlich konfligiere die diffuse ideologische Orientierung der Zeitschrift mit ihrer Gemengelage aus Motiven bündischer Jugendbewegung, Romantizismus, Sympathien für das bolschewistische Russland und Nähe zur Kamarilla um Kurt von Schleicher, für den scharf analytischen Geist Schmitts, der seine Lehre, auch qua Rekurs auf Heinz O. Zieglers Die moderne Nation von 1931, fortan gegen solche Adaptionen zu immunisieren getrachtet habe (154), für Breuer zu Recht

${ }^{9}$ Etwa Edith Hanke/Wolfgang J. Mommsen (Hg.): Max Webers Herrschaftssoziologie. Studien zu Entstehung und Wirkung, Tübingen 2001. 
ein weiterer Beleg für Schmitts mangelnde Berührungsangst vor dem Gedankengut jüdischer Autoren. Der Ring hingegen wurde eine Plattform v. a. für Schmitts konservative Schüler wie Ernst Forsthoff und Ernst Rudolf Huber, sodass das Periodikum eine Weile »als Hausorgan des Schmittianismus» (195) gelten konnte.

Ähnlich sieht Breuer eine "Schmittianisierung des Deutschen Volkstums" (200), doch bei allem Respekt für seine Forschungsleistung, der Hang, die Debatten und Meinungsverschiedenheiten bis in ihre Verästelungen nachzuzeichnen, strapaziert dann doch die Geduld des Lesers, eine Straffung hätte dieser Kapitelfolge gut getan, denn unbestritten ist ohnehin: "Carl Schmitt war kein General, der eine Truppe befehligte, sondern Mittelpunkt eines Kreises von akademisch geschulten, hochqualifizierten Intellektuellen, die durchaus fähig waren, ihre eigenen Wege zu gehen und dabei Konflikte mit ihrem Lehrer in Kauf zu nehmen« (213). Versöhnlich stimmen dagegen die von Breuer eingestreuten Kurzbiographien von Personen wie Waldemar Gurian, Wilhelm Grewe, Bernhard von Mutius u. a., die nachdrücklich illustrieren, wie Schmitt auf höchst verschiedene Charaktere aus sehr unterschiedlichen Milieus gleichermaßen faszinierend wirken konnte.

Eher tentativ mutet dagegen das auch als Ausblick fungierende Schlusskapitel an, da der selbstgewählte Zeitraum im Versuch, Oswald Spengler und Schmitts einsetzendes Spätwerk der esoterischen Großraumtheorie, die dieser allerdings noch immer als mit Nationalismus kompatibel erachtete, engzuführen, ${ }^{10}$ nun doch überschritten wird. Dies jedoch gelingt dem Autor nur allusiv - und es spricht für seine Lauterkeit, zu konzedieren: "Eine genauere Analyse zeigt jedoch, daß auch in dieser Phase die Unterschiede zu Spengler größer sind als die Gemeinsamkeiten« (269).

Insgesamt problematisch für das Buch bleibt der Begriff Intellektuellenpolitik, Breuer dünkt schon die unablässige Publikation von Essays, Broschüren, Stellungnahmen, im junghegelianischen Jargon: kritischen Antikritiken und "Korrespondenzwürstchen" (MEW 8: 313), sehr unschmittianisch eine genuine Form politischen Handels. Damit zeigt sich, so erhellend sie auch ist, die Schwäche aller Ideengeschichte, indem sie notwendig innerhalb des papiernen Kosmos verbleibt. Die doch dramatische Realhistorie der letzten Weimarer Jahre, als Folie für die Lektüre des Buches unverzichtbar, muss Breuer als bekannt voraussetzen, sie hallt lediglich gedämpft wider. Auch scheint nur punktuell auf - und dies gilt ebenso für Kervégans begriffliche Rekonstruktion -, wie wenig die Variation politischer Semantik letztlich mit dem faktischen Entscheidungsvollzug zu tun hat; etwa, wenn die Papen-Regierung ihre Bitte an

${ }^{10}$ Dies hat Florian Meinel: "Anatomie eines Nationalisten. Stefan Breuer versucht, Carl Schmitt zu stellen«, in: Zeitschrift für Ideengeschichte VII/2 (2013), S. 123-126, hier: 126, zu Recht betont. 
Schmitt, beim Ausarbeiten eines neuen Verfassungsentwurfs maßgeblich die Feder zu führen, kurzerhand wieder stornierte und damit den Geltungsbedüftigen düpierte (222). Unter dem NS-Regime, das ob der zeitlichen Beschränkung nur einige Streiflichter erreicht, habe es Schmitt versäumt, ein System von Bündnispartnern zu implementieren (230), weshalb er, so ist zu ergänzen, seiner Kompetenzbeschneidung 1936 nichts entgegenzusetzen hatte. ${ }^{11}$

Der Schmitt der Weimarer Jahre akzeptierte trotz aller Umbesetzungen oder Revisionen innerhalb der eigenen Theoreproduktion den modernen Begriff der Volkssouveränität $(60,143)$ und präferierte einen plebiszitär-autoritären Nationalstaat mit präsidialer Spitze, keineswegs stand er der Weimarer Reichsverfassung, das ist Breuer am wichtigsten, von vornherein ablehnend gegenüber (46, 68f., 102, 112, 122). Doch rächt es sich gerade bei diesem Resumée, dass Breuer, der auf z.T. irreführende angloamerikanische Forschung rekurriert (58), versäumt, zwischen dem emanzipativ-liberalen Risorgimento-Nationalismus und seiner aggressiv-integralen Spielart des späten 19. Jahrhunderts konsequent zu unterscheiden, sodass sich zumindest der unbedarfte Leser fragen mag, aus welchem Ressentiment sich Schmitts Hass auf den Liberalismus mit seiner für ihn irrigen Auffassung etwa der Souveränität des Rechts eigentlich speiste.

In Verteidigung gegen die terribles simplificateurs dem Werk Schmitts eine differenziertere Sicht angedeihen zu lassen und vorschnellen Verrechnungen einen Riegel vorzuschieben - hier trifft sich Breuer mit Kervégan -, ist nicht die geringste Absicht dieser manchmal zwar spröden, doch sehr nützlichen Studie. Wenn im Durchgang ebenso transparent wird, dass Schmitt kein Interesse an den "Ideen von 1914 « und der Kriegszieldebatte hatte, der nur binnen weniger Tage im November 1918 erfolgten Abdankung sämtlicher deutscher Dynastien mit Gleichmut begegnete, als PreuBen-Hasser alles Militärische verachtete und nichts vom Plan zur Restauration der Hohenzollernmonarchie hielt, wie sie im Papen-Kreis ventiliert wurde, ist Schmitts bemerkenswert eigenständiger Konservatismus als Residualphänomen, das wohl keine Kontextualisierung zur Gänze wird auflösen können, mit aller Deutlichkeit ins Bewusstsein gerückt.

\section{III}

Die Schmitt-Industrie hat mit Der Schatten Gottes den nun vierten umfänglichen Tagebuch-Band vorgelegt. Auch er fokussiert wieder einen wichtigen biographischen Abschnitt, die ersten Jahre der Bonner Profes-

11 Menring, a.a.O., S. $378 f f$. 
sur, doch völlig beherrschend ist hier das, was Reinhard Mehring treffend Schmitts »Liebesstaffette» genannt hat ${ }^{12}$.

So stand er zwischen vier Frauen: Paulina "Carita « Dorotić, der vorbestraften Diebin, Hochstaplerin und Noch-Ehefrau, die in München verblieb und v. a. über Geldforderungen und dem sich anbahnenden Scheidungsprozess präsent ist, der stürmischen Beziehung mit der irischstämmigen Australierin Kathleen Murray, der Schmitt en passant ihre bei Ernst Robert Curtius in Marburg, der das Spiel sehr wohl durchschaute, eingereichte Dissertation schrieb - "wie schön und anständig ist sie, jedes Wort von mir« (87) -, der Münchner Ärztin und Übergangsgeliebten Carola »Lo« Sauer und Duschka Todorović, der baldigen zweiten Gemahlin. Mitten in der Murray-Affäre: "Ich habe zum 2. Mal mit einer Frau zu tun und sehe, dass sich alles wiederholt. Das geht so bis in alle Einzelheiten, dass ich oft glaube, nun zum 2. Mal in demselben Examen zu sein. [...] Aber für einen Christen ist es verständlich, wenn ich sage: Es handelt sich bei jedem Examen darum, durchzufallen« (23). Carita wird vom zur Astrologie und Spökenkiekerei neigenden Schmitt mehr als einmal ernsthaft des Schadenzaubers verdächtigt, was ihn nicht davon abhält, sich periodisch nach ihr zurückzusehnen, Murray glühend besungen ("schönste Countess", 60), Sauer erweist sich als zu emanzipiert, sodass die Angelegenheit bald erkaltet, »ich bin glücklich, wenn es keinen Krach gibt« (137), Todorović dagegen nähert sich Schmitt behutsam, die "Bauernhaftigkeit ihres Wesens" (206) zieht ihn ganz so an wie ihr anfängliches Desinteresse an seiner Person.

Arbeitet man sich nun durch diese vielen hundert Seiten, so wird neben dem Umstand, dass Schmitts Liebesrhetorik, die, wären da nicht die Zitate aus der gesamten Weltliteratur, zuweilen anmutet, als schreibe ein entfesselter Pennäler, dreierlei deutlich. Zunächst ist sein superlativisches Vokabular fungibel, alle je aktuellen Geliebten werden mit sich sehr ähnlichen Kaskaden schmachtender Briefe, deren Wortlaut Schmitt im Tagebuch zur Sicherheit festhält, überzogen. Sodann ist es die wohl nur im katholischen Milieu so mögliche, blühende Faszination durch und Anfälligkeit für seltsam hybride, schwül-überzogene Metaphern und Motivkomplexe mit ihrer den Leser geradezu anspringenden Bildlichkeit, wie man sie seit Baudelaire kennt, "die Freude der Verdammnis« (406), in der sich Schmitt wähnt, wird schwelgerisch ausgekostet, wenn er sein erotisches Begehren ausagiert, die Vorliebe gerade für französische Dichter der Postromantik ist von daher alles andere denn zufällig: "Wie bin ich doch französisch" (163). Schließlich und frappant: So schwankend, wie sich sein Urteil über Kollegen und Bekannte ausnimmt, ist auch das über seine Frauen, an einem Tag sind sie entrückt, am nächsten erscheinen sie beschränkt, lächerlich eigensinnig und nervtötend - ad lib, sodass Schmitt hier als Inkarnation des Momentanismus erscheint, ein Charakterzug, den er beim romantischen

12 Reinhard Mehring: „Vom Kreuzweg des streuen Zigeuners.، Carl Schmitts Liebesstaffette«, in: Merkur 68 (2014), S. 1109-1112. 
"Occasionalismus" bekanntlich inkriminierte. Wenn der Verfechter des Dezisionismus eines nicht konnte, dann sich entscheiden.

Obwohl die Karriere nach dem Greifswalder Intermezzo in den »arktischen Fluren Pommeraniens" (10) nun an der Bonner Universität erkennbar Fahrt aufnimmt und Schmitt täglich mit Kollegen, Freunden, Liebschaften verkehrt, gibt er den "Buchhalter meiner Verzweiflung« (83). Der Leser erlebt den "Kronjuristen des Dritten Reiches" (Gurian) im so empfundenen Wartestand: "Sehnsucht nach wirklichem Leben, Geld zu verdienen, nach wirklicher Macht« (109); "mir gehört die Zukunft" (413), doch Schmitt ist geradezu zerscherbt von depressiven Verstimmungen, ständiger Müdigkeit und Angstzuständen, die man nur unter Vorbehalt Panikattacken nennen möchte, künden sie doch eher von einer metaphysischen Weltangst - was ihm keinesfalls entgeht: "Inzwischen ahne ich die Einsamkeit jedes Menschen, die unentrinnbare Isoliertheit, aus der einen auch keine Frau reißen kann" (180), er leidet unter einer "objektlose[n] Sehnsucht, deren Grund die Objektlosigkeit ist« (176). Dann aber wieder fühlt er sich "ganz frei von den Menschen. Welche Wonne« (250). Schmitt imaginiert sich in einer selbstverfertigten, literarisch keineswegs bemerkenswerten Legende als "treuer Zigeuner", der sein Weib auf dem Rücken zur päpstlichen Audienz schleppt (564-569), sieht sich als Kreuzfahrer, Robinson - und bevor man Gelegenheit hat zu befürchten, dass er auch noch eine Postfiguration Christi vornehmen wird, tut er's (402, 411, 416).

Nur als Antidot zu seinem Leiden am eigenen Momentanismus erklärt sich Schmitts minutiöses Aufnotieren nicht nur von konsumierten Weinflaschen, Schokoladentafeln und Schinkenbroten, sondern es geht hinab bis zu den »crescendofreien Alltagsrituale[n] «"13 der Qualität des Mittagsschlafs, der Onanie und noch den Freuden des An- und Umkleidens. Mitsamt seiner vagierenden Lektüre gibt allein dieses Protokoll dem rastlosen Geist Schmitts überhaupt Form: »In der Bibliothek herumgelesen, das gehört zu meinem Sicherheitsgefühl« (192).

Sein Tagebuch ist ihm der einzige Ort der Selbsthabe, passend zu Mehrings Feststellung, dass Schmitt seit der Bonner Zeit schlechterdings jeden Beleg, jede Aufzeichnung archivierte und stellenweise untereinander zu vernetzen trachtete. ${ }^{14}$ Schmitt konnte, das sehen auch die Herausgeber (III), offenbar allein schreibend über sich verfügen, Selbstbewusstsein und -Gewissheit ereignet sich nur auf diesen manisch gefüllten Seiten. Ich schreibe, also bin ich, hier präsentiert sich ein Individuum, das befürchtet, sich ohne das tägliche Notat, noch den geringsten aufbewahrten Zettel, nicht empfinden zu können, sondern sich verlieren zu müssen.

13 Für Hans Ulrich Gumbrecht: Diesseits der Hermeneutik. Die Produktion von Präsenz, übers. v. Joachim Schulte, Frankfurt/M. 2004, S. 157, nicht zufällig Momente der »Präsenzkultur».

${ }^{14}$ Mehring: Carl Schmitt, a.a.O., S. 566. 
Auch auf Schmitts Antisemitismus wirft der Band neues Licht. Hielt er ihn selbst für aus mythologischen Wurzeln gespeist, so wird doch deutlich, dass Schmitt sich unter den zahlreichen Fachkollegen der zweiten jüdischen Assimilation als Parvenu vorkam. Er litt unter seiner kleinbürgerlichen Herkunft, seinem roten Gesicht, dem vermeintlich proletarischen Gang und seiner ihm peinlichen Begeisterungsfähigkeit, "Angst vor diesem gescheiten Juden « heißt es an einer Stelle (196), und es ist nicht ganz klar, ob sich Schmitt nur auf einen Text Carl Landauers oder gar auf seinen Lektor bei Duncker \& Humblot, Ludwig Feuchtwanger, bezieht, zu dem doch ein wenigtens minimales Vertrauensverhältnis bestehen sollte. Erich Kaufmann, dem er später in Berlin noch übel mitspielen sollte, verachtet er von Anbeginn, gegen Albert Hensel, aus namhafter Familie stammend, entwickelt er fast körperlichen Widerwillen, was ihn nicht hindert - auch das lehren die Tagebücher - privat sehr wohl mit Juden zu verkehren, Georg Eisler war in dieser Zeit noch ein enger Freund (393).

Es ist eine wahnwitzige Epoche, Geldentwertung, Ruhrkampf, die Ermordung Rathenaus, der Hitler-Putsch hört noch auf den Namen Ludendorffs, Bonn liegt in der französischen Besatzungszone, weshalb man bei Fernreisen eine Stunde Zeitverschiebung zu berücksichtigen hat; im subjektiven Reflex ist alles da, ein Fremdenführer in Wetzlar "macht Gedichte für eine Metzgerinnung und bekommt dafür Würste " (72), Schmitt erhält am 31. Oktober 1923 "fast 3 Billionen" Reichsmark an Bezügen (263), spekuliert ein wenig an der Börse und nutzt wie alle anderen auch die Hyperinflation, um seiner Schulden ledig zu werden. Während der Bürgerkriegsstimmung der frühen Zwanziger Jahre sieht er bald »wieder Banden durch Deutschland ziehen" (142), Paul Ludwig Landsberg, in dessen Elternhaus Schmitt verkehrt, wird Hohn zuteil: "Dachte daran, wie weich diese Leute sind, und der Junge ist Kommunist. Er denkt nicht daran, auf seinen Reichtum zu verzichten. Die einzige Konzession ist, dass er sich die Hose nicht bügelt. Großer Gott« (193).

Dennoch lebt Schmitt, trotz permanenter Lamenti, mit seinen häufigen Reisen, täglichen Café- und Restaurantbesuchen, recht behaglich, "schöner Kaffee, und ein Stück Kuchen und Völkerrecht«(79), heißt es zu Beginn der Bonner Lehrtätigkeit, die Güte seiner Veranstaltungen, die er auch schon mal aus dem Stegreif abhält, bewertet er nach ästhetischen Kriterien (»schön gesprochen«), doch bald nimmt ihn der universitäre Betrieb in die Pflicht, sodass nun auch die Klagen über Doktorexamina mit unklugen Kandidaten und Fakultätssitzungen nicht mehr abreißen. Namhafte Bonner Schüler wie »Gurian, ein russischer Jude aus Köln« (170) und Forsthoff tauchen erst spärlich auf, über die Entstehungsprozesse seiner Schriften aber - in die hier relevante Zeit fallen so wichtige Texte wie Römischer Katholizismus und politische Form, der Beitrag zur Weber-Erinnerungsgabe und Die geistesgeschichtliche Lage des Parlamentarismus - erfährt der Leser herzlich wenig, mal wird Korrektur gelesen, mal gehen Schmitt Sonderdrucke zu, die vielgerühmte Politische Theologie wurde geschrieben, „teils als ich im Bett mit K. lag, teils während sie nackt auf meinem Schoß saß« (429).

Daher liegt hier kein Arbeitsjournal vor, sondern wahrhafte journeaux intimes, die Selbstbefragung einer zutiefst unsicheren Existenz. Es mag da- 
zukommen, dass für Schmitt das protestantische Pathos von »Wissenschaft als Beruf keineswegs so verpflichtend war wie für Max Weber, gerade der Spezialisierungsforderung konnte er sich nicht fügen. Schmitt notiert größtenteils die Lektüre von Weltliteratur inklusive ihrer eher abseitigeren Autoren: "Diesen geistigen Luxus brauche ich, ich kann ihn nicht entbehren" (245), nur sehr punktuell ist die Auseinandersetzung mit eigentlicher Fachliteratur zu beobachten und Hegel, den mancher für Schmitts zentralen Gesprächspartner nimmt, ist ihm »der summus deceptor", großer Betrüger (49).

Erst der dritte Teil des Bandes gibt zumindest stellenweise eine Art Brouillon von Ideen, etwa zu Repräsentation, Dezisionismus, Ordnung und Form, Klassenkampf und Nationalismus (395, 400, 420), das aber über Stichworte, die sich sämtlich in seinen Schriften ausgeführt finden, nicht hinausreicht. Gelegentlich, im Hohn über das "Philosophensystem der Klubsessel" (451) oder Goethe und »die stinkende Bildung der Inselbücherei« (403) schlägt noch der Bohèmien durch, doch vor der Folie Nietzsches und Kierkegaards (447) konfligiert hier scharf der "Glaube an das Leben" (445) mit nihilistischen Einlassungen, das ist die Grundmelodie: "Das Nichts des Lautlosen öffnet sich und gebiert einen Ton aus, verschlingt den Ton wieder in sein Nichts, das sich zurückstürzt, als sei es vor dem Schall seines Wesens entsetzt. - Nun wird es wieder still« (428).

Nicht bestritten sei, dass manchmal ein Aphorismus in der Tradition des französischen Moralismus gelingt: "Es geht so dumpf zu in der Welt, dass gelegentlich sogar die Klugheit etwas auszurichten vermag« (496), doch auch an Kryptik ist kein Mangel: "Klassischer Geist: Kreis und Kugel; aus der Überwindung klassischen Geistes wird Amerika entdeckt, dadurch aber wird erst die Kugelgestalt der Erde klar, und es stellte sich also heraus: die Erde ist wirklich eine Kugel" (420). Ganz überwiegend hadert Schmitt mit Gottferne und -Nähe, »ich verberge mein Haupt im Schoße einer Frau, um das Medusenhaupt der Zeit nicht zu sehen; es ist alles umsonst« (429) - wer wollte da widersprechen? In derselben Zeile ist Gott "durchsichtig« und wirft doch einen Schatten (396), deutlicher kann sich Schmitts Schwierigkeit mit der Entscheidung wohl nicht artikulieren. ${ }^{15}$

Jeder, der einmal an der Edition einer Brief- oder Tagebuchausgabe mitgetan hat, weiß, welch Mühe die Rekonstruktion von längst verschollenen Personen, Pensionen und Restaurationen, die oftmals nur über Umwege gelingt, bereitet. Die Herausgeber haben, ganz abgesehen von der Kärrnerarbeit der Transkription fast durchweg stenographierter Einträge, die der bereits verstorbene Hans Gebhardt besorgte, insofern hervorragende Arbeit geleistet, eher selten konnte etwas nicht ermittelt werden.

15 Menring: „Vom Kreuzweg«, a.a.O., S. 1109, macht auf die Herkunft des Motivs aus den Psalmen aufmerksam. Konkret alludiert ist Ps. 121, 5. 
Daher nur ganz wenige Korrekturen und Ergänzungen: Clemens Brentanos Godwi ist kein "Gedicht" (554), sondern sein einziger Roman, Guy de Maupassants »Horla", den Schmitt im Original gelesen haben dürfte, ist keine "Novellensammlung " (89), sondern eine ziemlich subtile Schauergeschichte, die Schmitt ausgerechnet im psychisch desolaten Zustand identifikatorisch liest und prompt von der Angst, wahnsinnig zu werden, ergriffen wird, denn das Notat der Folgenacht könnte aus dem Text selbst stammen: „Es wird täglich schlimmer. Wie lange noch?« (ebd.). Ähnlich verhält es sich mit dem adaptierten »Flügelschlag des Irrsinns « (140), den schon Baudelaire am 23. Januar 1862 über sich hinwegstreichen fühlte, doch behält Schmitts Liebäugeln mit dem Wahnsinn etwas Kokettes. Mehr noch, ein "Schluck aus dieser Flasche macht mich frei« (403) bezieht sich, obwohl kein Originalzitat, offenbar auf Fausts zweiten Entgrenzungsversuch als Selbstmord via eines beherzten Schlucks aus der Opiumphiole (Faust I, V. 687ff.), da die Notate des Tages von Goethelektüre zeugen. Imaginierte der Katholik Schmitt, der sich hier als verzweifelter Gottsucher präsentiert, gar den Suizid?

Es bleibt die Frage, ob wir all das in solch detaillierter Extension wissen müssen. Unbedingt, sagen die Herausgeber, Schmitt sei schließlich »ein Fall der modernen Kulturgeschichte«(III), doch an die großen existenzialistischen Tagebücher Kafkas oder Cesare Paveses reicht Schmitts Reflexion eben doch nicht heran. Gewiss, »1921 bis 1924 wurde Schmitt der Autor, den wir kennen «, ${ }^{16}$ doch belastbare Bausteine $\mathrm{zu}$ einer spezifisch intellektuellen Biographie lassen sich im privatistischen Geröll dieser Notate nur vereinzelt ausmachen, zumal Autoren wie Mehring und Breuer das Notwendige schon aus ihnen schöpfen konnten. Dass sich nach jahrhundertelanger Durststrecke und der Suppression des idealistischen 19. Jahrhunderts, das auch eines des glühenden Protestantismus war, mit Heidegger, Scheler und eben auch Schmitt überhaupt wieder ein originäres Denken aus dem Katholizismus heraus regt, ist das wohl eigentlich Erstaunliche. Kaum noch von der Hand zu weisen ist jetzt freilich ebenfalls: Deutschlands berühmt-berüchtigster Staatsrechtler, er war ein Freak.

Ingo Meyer

Von der Recke-Straße 4

33615 Bielefeld

vdr1411@gmx.de

16 A.a.O., S. 1112. 